\title{
Evaluation of Lumber Spine Stenosis on Magnetic Resonance Imaging Correlating with Its Clinical Manifestation
}

\author{
Madiha Anwar* \\ Student, Superior College Lahore, University Campus, 17-KM Raiwaind Road, Kot Arain, Lahore, Pakistan \\ Rukhsar Shoukat \\ Student, Superior College Lahore, University Campus, 17-KM Raiwaind Road, Kot Arain, Lahore, Pakistan \\ Kainat Ramzan \\ Student, Superior College Lahore, University Campus, 17-KM Raiwaind Road, Kot Arain, Lahore, Pakistan \\ Mustansar Hussain \\ Student, Superior College Lahore, University Campus, 17-KM Raiwaind Road, Kot Arain, Lahore, Pakistan \\ Junaid Khalil \\ Student, Superior College Lahore, University Campus, 17-KM Raiwaind Road, Kot Arain, Lahore, Pakistan \\ Amna Babar \\ Lecturer, Superior College Lahore, University Campus, 17-KM Raiwaind Road, Kot Arain, Lahore, Pakistan \\ Rana Muhammad Athar Azeem Shams \\ Lecturer, Superior College Lahore, University Campus, 17-KM Raiwaind Road, Kot Arain, Lahore, Pakistan \\ Rana Muhammad Bakhtwar Khan Sajawal \\ Lecturer, Superior College Lahore, University Campus, 17-KM Raiwaind Road, Kot Arain, Lahore, Pakistan
}

\begin{abstract}
Lumber spine stenosis (LSS) is one of the major reasons of spinal surgery all over the globe. Despite using standard Diagnostic tools for LSS, the clinical and imaging findings often do not correlate. Over past years, the central spinal canal stenosis is assumed to be the diagnoses of lower back pain, while other anatomical stenosis (i.e lateral recess stenosis) not gaining as much attention. This fact assumed to be the reason for failed back surgery. In this context numerous studies in past have done. The aim of this study is to verify the clinical manifestation of LSS correlation to its Diagnostic find. To evaluate the lumbar spine stenosis on magnetic resonance imaging \& correlating with its clinical manifestations. The duration of study was three months. Study was done at Lahore General Hospital, Pakistan. A cross-sectional analytical study included 120 patients. According our study the patients comes with lumber spine stenosis at different levels. At L4L5 presents 39 (32.5\%) patients, 47(39.2\%) patients reveal at the level of L5S1, 14 (11.7\%) patients at L2L3, and only $3(2.5 \%)$ patients appear at the level of L1L2, 19 (15.8\%) patients show stenosis at L3L4 Then we correlate the pain intensity with all lumber spine levels and the results are $35(29.2 \%)$ patients are absent and $85(70.8 \%)$ presents at the level of L4L5. On the level of L5S1 pain intensity is absent in $81(67.5 \%)$ patients, \& present in 39 (32.5) patients. On the level of L2L3 106 $(83.5 \%)$ absent and $14(11.7 \%)$ patients are present pain intensity. On the level of L1L2 $117(97.5 \%)$ absent of pain intensity and only $3(2.5 \%)$ patients are present with pain intensity. The study concluded that the clinical manifestation of lumber spine stenosis to devise the management plan for the patient for better diagnoses in magnetic resonance imaging in LSS.
\end{abstract}

Keywords: Lumber spine stenosis, manifestation, MRI

DOI: $10.7176 / \mathrm{JHMN} / 90-10$

Publication date:June $30^{\text {th }} 2021$

\section{Introduction}

Lumbar spinal stenosis (LSS) is a situation that is medically demonstrated as constriction of spinal canal, lateral recess and neural foramina, with possible subsequent neural or vascular compression. The abnormal dimensions or pathological variations included isolation. it may also without or with related disk bulge or herniation. Also, it is able to be correlated with degenerative spondylolisthesis and scoliosis. (1) In the united country lumbar spinal stenosis (LSS) declared in greater than 200,000 adults. In the patients over 65 years it is a general reason for spinal surgery. (2) Vertebral Bodies of lumbar spine have some unique features, are deeper anteriorly than posteriorly, producing the lumbosacral angle. The vertebra has two basic parts: vertebral body \& posterior arch-that include pedicle and posterior elements. Between two vertebral bodies, there is an intervertebral disc composed of Annulus 
fibrosis and nucleus pulposus. The disc anatomical variation plays an integral role that lead towards the pathophysiology of LSS. (3) LSS is a pathology that originate mostly due to certain structural differences, on the basis of anatomical variations it can be divided into mono-segmental or multi-segmented, and bilateral or unilateral. The lumber spine stenosis can be classified as central, lateral or foraminal by observing anatomy. Determined by the pathology the stenosis can occurs alone on central, lateral and foraminal site. However, in combination Central canal stenosis may result from a reduced in the antero-posterior, transversal. In the case of hypertrophy of the facet joints, the flavum ligament. It is combined with the secondary diameter for loss of height of the intervertebral disc height with or without bulging of the intervertebral disc. Foraminal stenosis can occur due to the narrowing of the anteroposterior disc space and the overgrowth of the structures in front of the capsule of the facet joint. It can also occur perpendicularly when posterolateral osteophytes of the vertebral endplates protrude into the foramen with a fibrous ring that is laterally arched or herniated. this compresses the nerve root against the upper pedicle. The L45 discs are most commonly affected by LSS, followed by L3-4, L5-S1, and L1-2. In addition, the posterior joint in this region affords more rotation and are therefore more exposed to rotatory strain. (4) The typical dimensions of lumbar spine stenosis categorized as congenital/developmental, absolute, \& obtained lumbar spine stenosis. The study found that the absolute LSS was $2.6 \%$, the congenital relative LSS $4.7 \%$, the acquired absolute and relative LSS $22.5 \%$ and 7, respectively.3\% for sixty- and sixty-nine-years old population, the relative $47.2 \%$ and absolute LSS became $19.4 \%$. Congenital stenosis can also predispose a person with slight degenerative adjustment to grow to be symptomatic in advance in life.(5) It may additionally raise because of congenital malformation like spinal dysraphism (incomplete vertebral arch closure). In few cases, the affected person has developmental degenerative adjustment (Early vertebral arch ossification, shortened pedicles. Thoracolumbar kyphosis, apical vertebral wedges, anterior vertebral tips, etc. That increase the congenital narrowing of the vertebral canal. Canal additions that cause degenerative changes often cause central and lateral stenosis, include ossification of posterior longitudinal ligament, vertebral body (bone spurs), intervertebral disc, and epidural fats. The pathology often results from acquired degenerative changes i.e. spondylosis. Degenerative modifications normally motive relevant and lateral stenosis; ligament flavum hypertrophy, side hypertrophy, and disc bulge. Any form of adjustment might also additionally be the number one reason, despite the fact that in relevant stenosis, the ligament flavum normally is the first-rate contributor. LSS incidence have become expanded through age, about $1.7-2.2 \%$ in 40 -forty 9 years vintage population, and $10.3 \%-11.2 \%$ in $70-79$ years older population. Another examine stated that the prevalence of symptomatic LSS is $10 \%$. The LSS is the maximum purpose for >sixty-five years antique sufferers to go through the lumber spinal surgery. The prevalence of LSS with age is also controversial, depending on the gender of the patient. The prevalence of LSS in men hardly changes with age over 70, but the prevalence of LSS in women increases with age. (6) LSS has wide spectra of clinical presentation but the most classical clinical presentation associated with it is known as neurogenic claudication. The patients come with pain and discomfort they transmit. After a certain distance, the hips, thighs and calves will cause dysfunction and reduced walking ability. (7) In LSS patients, using some dynamic additives to sit or bend the lumbar spine can usually relieve pain symptoms. Strengthen the position of the lumbar spine and reduce the area of the spinal canal in the lumbar spine. (8) Patients with lumbar spine stenosis who suffer from lumbar spondylolisthesis, also comes with the symptoms of lower back pain including paresthesia, numbness in leg, tingling, imbalance and fatigue in lower extremity. Symptoms are typically bilateral and usually a-systematic. (9) MRI test of valuable stenosis typically suggest a circumferentially narrowed canal. MRI trial of important stenosis commonly recommend a circumferentially limited canal. Hypertrophic bone shows up as a darkish space of low indication of T1-weighted and T2-weighted pictures. Hypertrophy of ligamentum flavum shows up as a median sign on T1-weighted and T2-weightedimages, it additionally loss of fat in the epidural space because of delayed pressure as a deficiency of high T1 signal. (10) Lateral stenosis best seen on axial and sagittal perspectives as bone infringement and loss of fat sign (best perceive on T1-weighted pictures) encompassing the leaving nerve root. A sagittal view is created by using a field of view (FOV) of $32 \mathrm{~cm}$, matrix $512 \times 256$ and slice thickness of $4 \mathrm{~mm}$. The axial image is generated to utilize a FOV of $15 \mathrm{~cm}$, matrix $256 \times 320$ and cut thickness of $4 \mathrm{~mm}$. MRI arrangements/ sequences were sagittal T1 weighted spin echo repetition time (ms) echo time (ms) 500-600/12-17], T2 weighted picture (3500/120), axial T2 weighted turbo spin echo (3000-4000/60-150), T2 STIR (2500/60), and T1 turbo spin echo (600-700/10-15). (11)

\subsubsection{Results}

In this cross-sectional analytical study through Convenient sampling technique, we have selected total 120 patients, the minimum age of the patients included in the study was about 19 and maximum age was around 83 with the common standard deviation to be around 14.77 .

\subsubsection{Discussion}

According to our results the lumber spine stenosis was recorded to be maximum around and minimum to be around among 120 patients. Sameer Kitab study that were results, Age helped to explain only $7.8 \%, 1.2 \%$, and $1.9 \%$ of the variance in TDSS at L3-4, L4-5, and L5-S1, with weak positive and $9.6 \%$ and $12.2 \%$ of the variance in total 
degenerative spinal stenosis at L1-2 and L2-3 with a moderate positive correlation. (12)

Age has constantly a problematic characteristic of lumber spine stenosis, according to our research statistics we have divided the case frequency of lumber spine stenosis from 19-25 to 75-83, in which the minimum age recorded of the lumber spine patient was found to be around 19 year and maximum age of the lumber spine patient was recorded to be around 83 years of age. And according to the age group-based frequency distribution of lumber spine stenosis patients, the most frequent number of cases was reported from the age group 65-72.

Pain is always a starting sign for a disease. In our study when we compare the gender with pain intensity the females are $35(35.4 \%)$ are bearing pain in lumber spine stenosis, 15 females (16.4\%) are having pain free lumber spine stenosis. Also, when it comes to males 20 (20.4\%) did not feel any pain but $50(49.6 \%)$ suffering from pain. Our study shows that the males are tolerate more pain intensity as the females. In a study 79 clinicians from 29 countries, with an average of $19( \pm$ SD: 12) years in exercise participated. The top sign was "leg or buttock pain even as walking", "flex ahead to alleviate symptoms towards leg. sense remedy while the usage of a purchasing cart or bicycle", "motor or sensory disturbance even as walking. (13)

Tingling is also a major sign that was seen during our study in the clinical history, our results shows that 21 females are feel tingling but 29 did not feel it at all out of total 50 females. On other side 41 males comes with tingling and 29 did not, out of 70 men patients.

Our study includes the patients comes with the disturbed gait that are 23 females and 27 females are having lumber spine stenosis but normal gate. On other side the 37 males have disturbed gait and 33 are represents with normal gait. Nagai conducted a study the results were shows that walking difficulty during the test increased with the maximum walking difficulty. In a study results shows, walking difficulty during the test increased from 4 (interquartile range, 1-5) to 9 (interquartile range, 7-10). The postural and Gait distance increased after the onset of maximum walking difficulty. (14)

During our study the patients comes with lumber spine stenosis at different levels. At L4L5 presents 39 $(32.5 \%)$ patients, $47(39.2 \%)$ patients reveal at the level of L5S1, $14(11.7 \%)$ patients at L2L3, and only $3(2.5 \%)$ patients appear at the level of L1L2, $19(15.8 \%)$ patients show stenosis at L3L4. Sumishia Orita were studied that, The L5 S1 radiculopathy with greater range of occurrence than any other lumbar levels as a result of anatomical factors. A study shows that the L5-S1 foraminal stenosis is greater in number, because of its anatomical and functional junction. $1-15 \%$ of all disc herniation due to reduce L5- S1 height. (15)

Then we correlate the pain intensity with all lumber spine levels and the results are $35(29.2 \%)$ patients are absent and $85(70.8 \%)$ presents at the level of L4L5. On the level of L5S1 pain intensity is absent in $81(67.5 \%)$ patients, \& present in 39 (32.5) patients. On the level of L2L3 $106(83.5 \%)$ absent and $14(11.7 \%)$ patients are present pain intensity. On the level of L1L2 117 (97.5\%) absent of pain intensity and only $3(2.5 \%)$ patients are present with pain intensity. On the level of L3L4 pain intensity is preset in $19(15.8 \%)$ patients and absent in 101 $(84.2 \%)$ patients.

\subsubsection{Conclusion}

The study concluded that the clinical manifestation of lumber spine stenosis to devise the management plan for the patient for better diagnoses in magnetic resonance imaging in LSS. LSS is a clinic \& radiologic disease with complicated relationships among of radiologic stenosis disclosed with the aid of using MRI and clinal manifestations. Lumber spine stenosis with inside the clinical manifestation displays the greater degree of morphologic.

\section{References:}

1. A comparative study of three conservative treatments in patients with lumbar spinal stenosis: lumbar spinal stenosis with acupuncture and physical therapy study (LAP study). Hiroyuki Oka, Ko Matsudaira, Yuichi Takano, Daichi Kasuya, Masaki Niiya etal,. s.l. : BMC Complementary Medicine and Therapies, 2018.

2. Management of lumbar spinal stenosis. Jon Lurie, associate professor1, Christy Tomkins-Lane, associate professor2. s.1. : The BMJ , 2016.

3. The Lumbar Neural Foramen and Transforaminal Epidural Steroid Injections: An Anatomic Review With Key Safety Considerations in Planning the Percutaneous Approach. Jacob C. Mandell1, Gregory J. Czuczman1, Glenn C. Gaviola1,etal. s.l. : AJR American journal of roentgenology , 2017.

4. Degenerative Lumbar Spinal Stenosis. Claudius Thomé, Prof. Dr. med., ${ }^{*}, 1,4$ Wolfgang Börm, etal,. s.l. : Deutsches Ärzteblatt international, 2008.

5. Predictors of Treatment Choice in Lumbar Spinal Stenosis: A SPORT Study. Mark F. Kurd, MD,1 Jon D. Lurie, MD, etal,. s.l. : HHS Public Access, 2018.

6. Prevalence of symptomatic lumbar spinal stenosis and its association with physical performance in a population-based cohort in Japan: the Wakayama Spine Study. Y.Ishimoto $\uparrow$ N.Yoshimura $\ddagger$ S.MurakiłH.Yamada etal. s.1. : Osteoarthritis and Cartilage, 2012.

7. Characteristics and determinants of clinical symptoms in radiographic lumbar spinal stenosis in a tertiary 
health care centre in sub-Saharan Africa. Marie Doualla-Bija, Mbeng Ashu Takang, Emmanuella Mankaa, Jude Moutchia, Pierre Ongolo-Zogo \& Henry Luma-Namme. s.l. : BMC, 2017.

8. Midsagittal Anatomy of Lumbar Lordosis in Adult Egyptians: MRI Study. Abdelmonem A. Hegazy1 and Raafat A. Hegazy, etal,. s.l. : Anatomy research international, 2014.

9. Clinical correlation of a new practical MRI method for assessing central lumbar spinal stenosis. H-J Park, S S Kim, Y-J Lee, S-Y Lee, N-H Park, Y-J Choi, etal. s.l. : The British Journal of Radiology, 2014.

10. Magnetic Resonance Imaging of the Lumbar Spine in People without Back Pain. Maureen C. Jensen, Michael N. Brant-Zawadzki, Nancy Obuchowski, et al. s.l. : The new england journal of medicine , 1994.

11. Relationship Between Magnetic Resonance Imaging Findings and Clinical Symptoms in Patients with Suspected Lumbar Spinal Canal Stenosis: a Case-control Study. Hadi Majidi, 1 Misagh Shafizad,2 Fatemeh Niksolat, et al,. s.l. : ACTA Actainformatica Medica, 2019.

12. Redefining lumbar spinal stenosis as a developmental syndrome: does age matter? Sameer Kitab MD1, Ghaith Habboub MD2, Salam B. Abdulkareem MD1, Muthanna B. Alimidhatti MD1, and Edward Benzel MD2, et al. s.l. : JNS Journal of Neurosurgery, 2019.

13. Consensus on the clinical diagnosis of lumbar spinal stenosis: Results of an International Delphi Study. Christy Tomkins-Lane, PhD, Markus Melloh, MD, PhD, Jon Lurie, et al. s.l. : HHS Public Access, 2016.

14. Quantification of Changes in Gait Characteristics Associated With Intermittent Claudication in Patients With Lumbar Spinal Stenosis. Nagai, Koutatsu PT, PhD*, $\dagger$ and Aoyama, Tomoki MD, PhD $\dagger$ et al. s.l. : Clinical Spine Surgery, 2014.

15. Lumbar foraminal stenosis, the hidden stenosis including at L5/S1. Sumihisa Orita, Kazuhide Inage, Yawara Eguchi, Go Kubota, et al. s.l. : Springer link, 2016.

\section{Gender * H/O Lumber spine stenosis:}

Table No 1: $\quad n=120$

Age

\begin{tabular}{lll}
$\mathrm{N}$ & Valid & 120 \\
\cline { 2 - 3 } Mean & & 0 \\
\hline Median & & 53.67 \\
\hline Mode & 54.00 \\
\hline Std. Deviation & & 72 \\
\hline Minimum & 14.770 \\
\hline Maximum & & 19 \\
\hline Percentiles & 25 & 83 \\
\hline & 50 & 42.00 \\
\hline & 75 & 54.00 \\
\hline
\end{tabular}

Table 1: Descriptive statistics of age 


\section{Chart 1: Age \& Frequency bar chart}
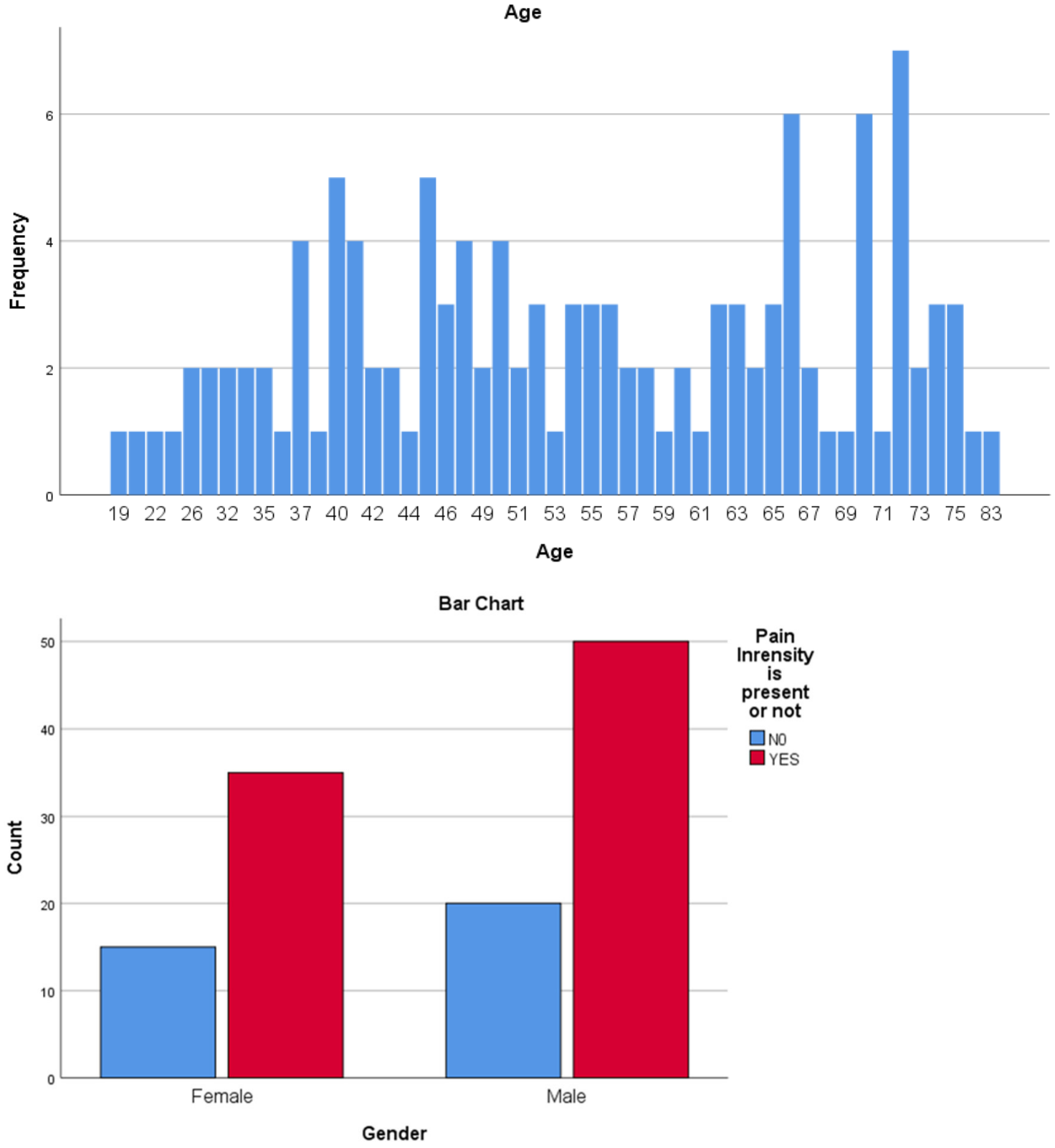
Chart 2: gender \& pain intensity bar chart

Bar chart 3: Stenosis level of lumber spine
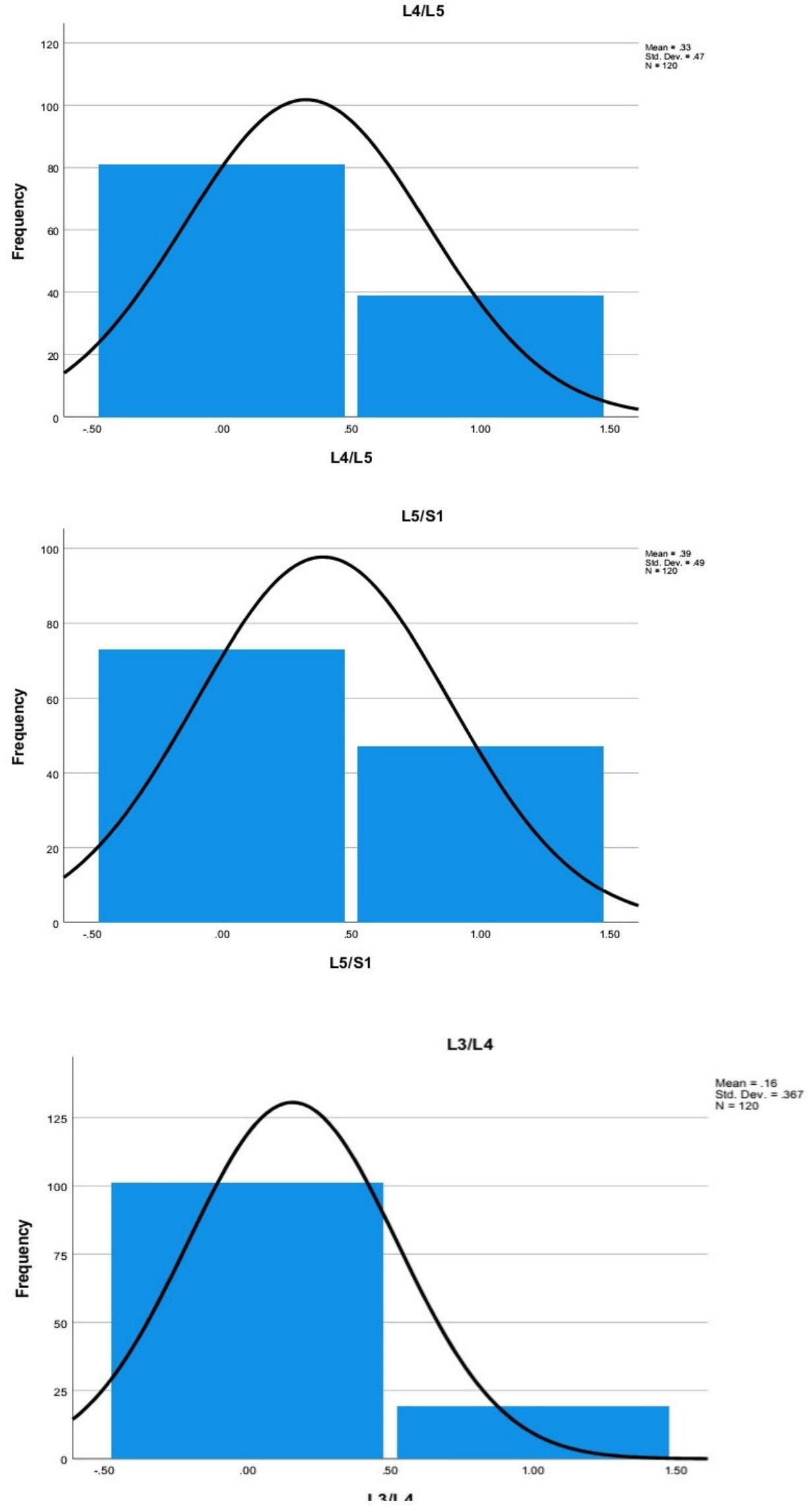

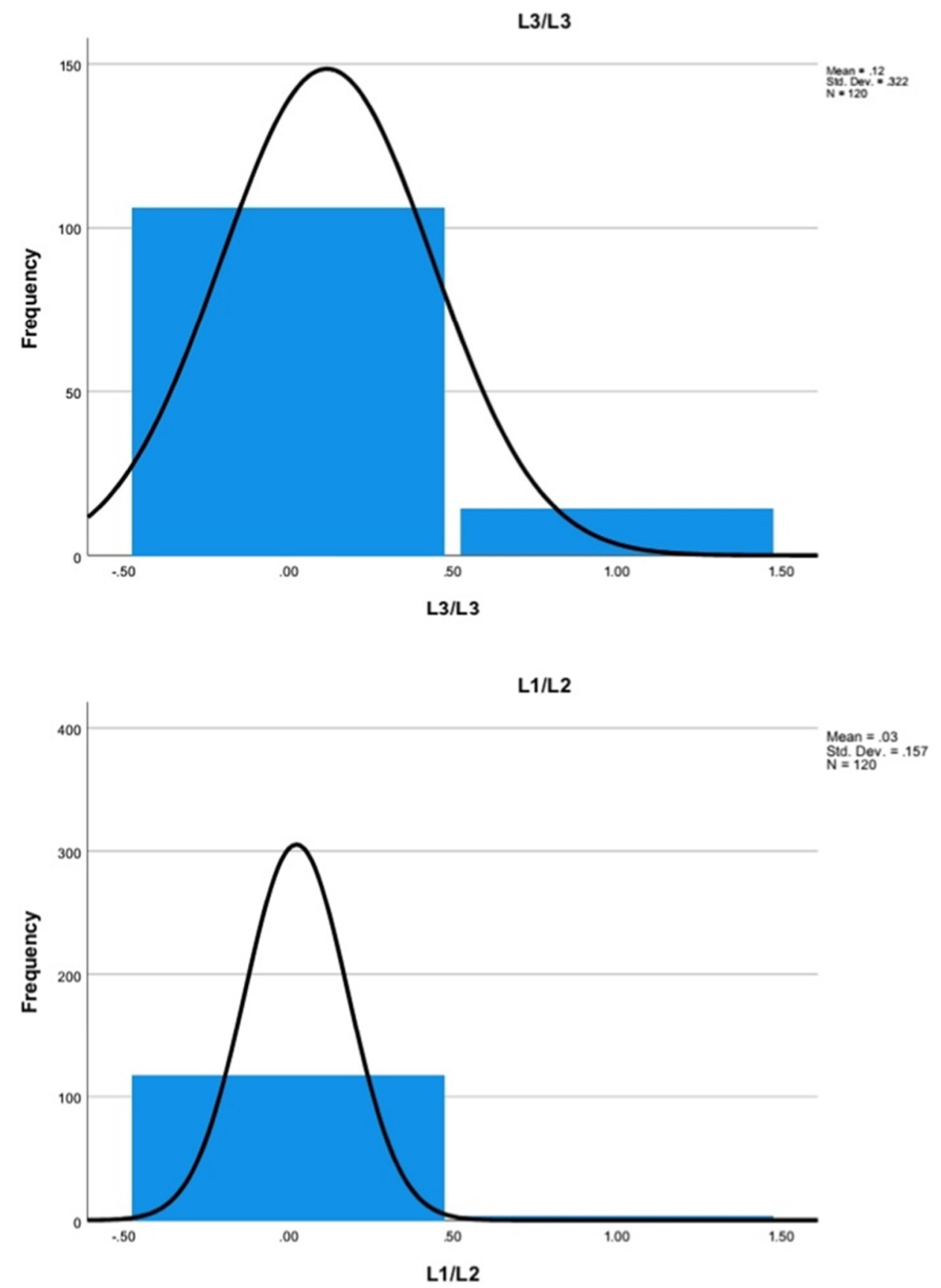
Figure 1:

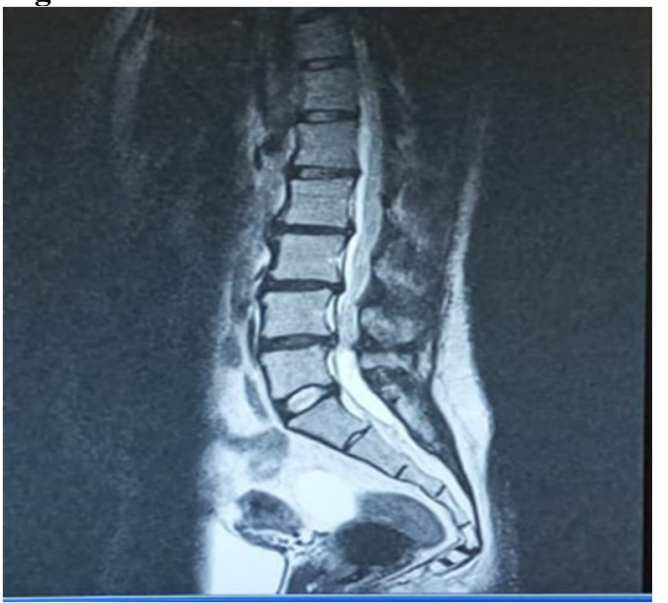

MRI T2 sequence Appearances consistent with L4/5 disc extrusion along with leg pain and tingling. Moderate descending $\mathrm{L} 4$ nerve root impingement also seen at $\mathrm{L3} / 4$ related to disc.

Figure 2:

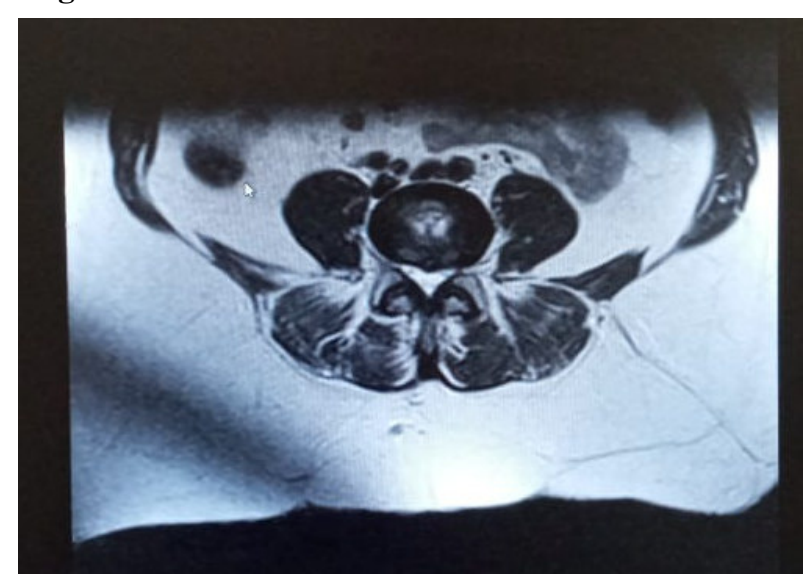

Right Para central disc extrusion at L5/S1 on a background of post right spinal laminectomy changes.

Figure 3:

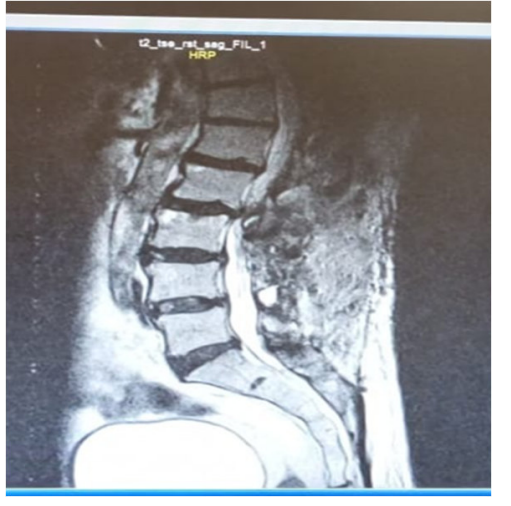

Appearances consistent with multilevel mild to moderate spinal stenosis with most marked changes at L2/3 and L5/S1 levels with disc-osteophyte related exiting and descending nerve root. 